\section{Hipercalcemia, peak monoclonal y leucemización como presentación de un linfoma de células $B$ de alto grado, con reordenamiento de MYC, BCL-2 y BCL-6 (Triple Hit). Caso clínico}

\author{
CAMILA PEÑA ${ }^{1}$, MARÍA JOSÉ MARTI²,a \\ PABLO VILLEGAS ${ }^{3}$, MARÍA SOLEDAD UNDURRAGA ${ }^{1,2}$
}

\section{Hypercalcemia, monoclonal peak and leukemic presentation of a High-grade B-cell lymphoma with MYC, BCL-2 and BCL- 6 translocations (triple hit). Report of one case}

High-grade B-cell lymphomas with rearrangement of MYC, BCL-2 and/or
CL-6 were introduced by the update of the WHO classification of lymphoid
eoplasms. They usually present unique morphological and molecular charac-
eristics, with an aggressive clinical outcome and worse prognosis. We report a
8 year-old female patient presenting with B symptoms and enlarged lymph
odes. Blood count showed pancytopenia and peripheral blood smears showed
arge lymphoid cells, some with nuclei and vacuoles. LDH was $3524 \mathrm{~g} / \mathrm{L}$ and
serum calcium was $11.5 \mathrm{mg} / \mathrm{dL}$. Flow cytometry immunophenotyping showed
pathological mature B lymphocytes. Protein electrophoresis showed a slight
monoclonal peak. The biopsy disclosed a triple expressor diffuse large B-cell
mphoma, arising from germinal center. FISH was positive for MYC, BCL-2
nd BCL-6 (triple hit) with a clonal evolution. Conventional cytogenetics showed
complex karyotype. Chemotherapy was started with R-CHOP (Rituximab/
cyclophosphamide/doxorubicin/vincristine/prednisone). She developed impaired
consciousness; the brain CT scan showed a large brain mass. The patient died
ithin 3 weeks.

(Rev Med Chile 2017; 145: 1485-1489)

Key words: Lymphoma, Large B-Cell, Diffuse; Proto-Oncogene Proteins c-bcl-6; Proto-Oncogene Proteins c- bcl-2.
'Unidad de Hematología Clínica. Sección de Hematología. Hospital del Salvador. Santiago, Chile. 'Laboratorio citogenética, Hospital del Salvador. Santiago, Chile.

${ }^{3}$ Servicio de Anatomía Patológica, Hospital del Salvador. Santiago, Chile.

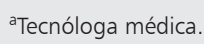

Los autores no declaran conflictos de interés.

Trabajo no recibió financiamiento.

Recibido el 19 de julio de 2017, aceptado el 7 de noviembre de 2017.

Correspondencia a: Dra. Camila Peña O. camipena@gmail.com

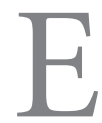

1 linfoma de células $\mathrm{B}$ de alto grado, con reordenamiento de MYC, BCL-2 y/o BCL6 (también llamados doble o triple hit), constituye una reciente entidad introducida en la revisión de la clasificación de neoplasias linfoides de la Organización Mundial de la Salud (OMS) de 2016 ${ }^{1}$. Suelen presentar características morfológicas y moleculares intermedias entre linfoma de Burkitt y linfoma difuso de células grandes $\mathrm{B}$, con clínica agresiva y peor pronóstico.

Se presenta caso de triple hit (TH) diagnosticado en el Hospital del Salvador, utilizando técnicas de citogenética convencional e hibridación in situ (FISH) en tejido, que se presentó con hipercalcemia, peak monoclonal y leucemización. 


\section{Caso clínico}

Paciente de 48 años, peruana. Comenzó en septiembre de 2014 con aumento de volumen cervical. En febrero de 2015 refirió compromiso del estado general, fiebre, sudoración nocturna y baja de peso de $5 \mathrm{~kg}$. En marzo de 2015 comenzó con aumento de volumen axilar e inguinal, disfonía y disfagia. Además, presentó tos seca irritativa. Finalmente, por mayor compromiso, con performance status de 3, consultó en nuestro centro.

Al examen físico destacaron adenopatías cervicales de $5 \mathrm{~cm}$, supraclaviculares izquierdas de $2 \mathrm{~cm}$, axilares bilaterales de $3 \mathrm{~cm}$, inguinales pequeñas y crurales.

En el hemograma destacó $\mathrm{Hb} 7,5 \mathrm{~g} / \mathrm{dL}$, leucocitos de $3.100 \mathrm{~mm}^{3}$ y plaquetas de $22.000 \mathrm{~mm}^{3}$. Al frotis de sangre periférica (SP) se vio células linfoides grandes, algunos con núcleo con hendidura, otros con vacuolas (Figura 1). VHS $70 \mathrm{~mm} / \mathrm{h}$, LDH $3.524 \mathrm{~g} / \mathrm{L}, \mathrm{B} 2$ microglobulina de $5,12 \mathrm{mg} /$ $\mathrm{dL}$, calcio 11,5 mg/dL. VIH, VHB y VHC fueron negativos. Electroforesis de proteínas mostró un pequeño peak monoclonal en beta gamma. IgA 77,5 (70-400 mg/dL), IgG 311,4 (700-1.600 mg/ dL), IgM 42,9 (40-230 mg/dL).

La tomografía computarizada (TAC) de cuello-tórax-abdomen y pelvis mostró adenopatías en todos los niveles del cuello, hasta $3,7 \mathrm{~cm}$, derrame pleural bilateral, masas hasta $2,8 \mathrm{~cm}$ pretraqueal, en hilio derecho y una masa que comprimía la tráquea al igual que ambos bronquios fuentes. Adenopatías axilares hasta $3,5 \mathrm{~cm}$, retroperitoneales hasta $3,6 \mathrm{~cm}$, cadena iliacas hasta $3 \mathrm{~cm}$ e inguinales de $1,5 \mathrm{~cm}$. Se realizó mielograma que mostró linfocitos patológicos, similares a los observados en la periferia. Inmunofenotipo por citometría de flujo de médula ósea (MO) mostró linfocitos patológicos maduros de estirpe B, con expresión de CD45 intenso, positividad para CD19, CD20, CD79a, IgM citoplasmática. Tdt negativo. Se realizó citogenética convencional por bandeo $\mathrm{G}$ en muestra de $\mathrm{MO}$, mostró un cariotipo complejo con alteraciones numéricas y estructurales (Figura 1). La biopsia de médula ósea resultó con infiltración en prácticamente $100 \%$. La biopsia de ganglio mostró linfoma no Hodgkin difuso de células grandes $\mathrm{B}$, de origen centro germinal, triple expresor, con CD10 positivo $100 \%$, MYC positivo en $50 \%$, BCL- 6 positivo en $30 \%$ y BCL2 positivo en $100 \%$ por inmunohistoquímica (IHQ). Ki 67 de 90\% (Figura 2). Se realizó FISH con sonda tipo BA Vysis para MYC, BCL-2 y BCL6 en muestra de ganglio linfático fijado en formalina y embebido en parafina. El análisis de MYC mostró múltiples patrones con 1 hasta 5 señales rojas; 1,2 y 4 señales verdes y 1 hasta 5 fusiones (Figuras 3 y 4). Fue interpretado como positivo para reordenamiento de MYC con amplificaciones del gen. Para BCL-2 y BCL-6 se observó patrones similares, concluyéndose linfoma de células $\mathrm{B}$ de alto grado, con reordenamiento de MYC, BCL-2 y BCL-6 (Triple HIT) con evolución clonal. Para descartar molecularmente la t $(8 ; 14)(\mathrm{q} 34 ; \mathrm{q} 32)$ del linfoma de Burkitt (LB), se realizó FISH para la $\mathrm{t}(8 ; 14)(\mathrm{q} 34 ; \mathrm{q} 32)$, resultando negativa. Se inició prefase con corticoides y luego quimioterapia con R-CHOP. Hizo reacción adversa severa a

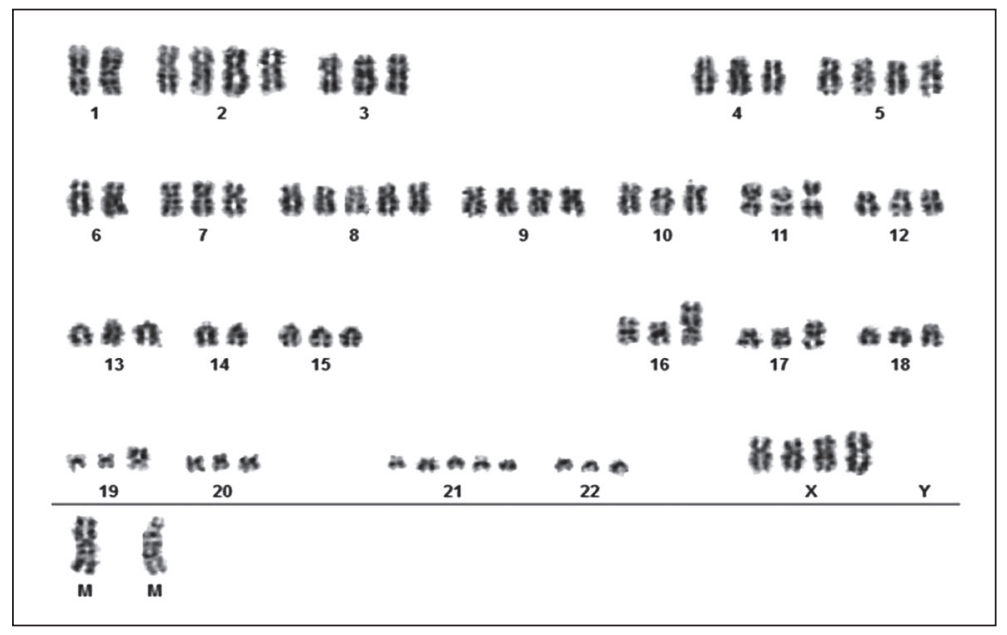

ura 1. Citogenética convencional complejo. 
CASO CLÍNICO

Hipercalcemia, peak monoclonal y leucemización en un linfoma triple hit - C. Peña et al
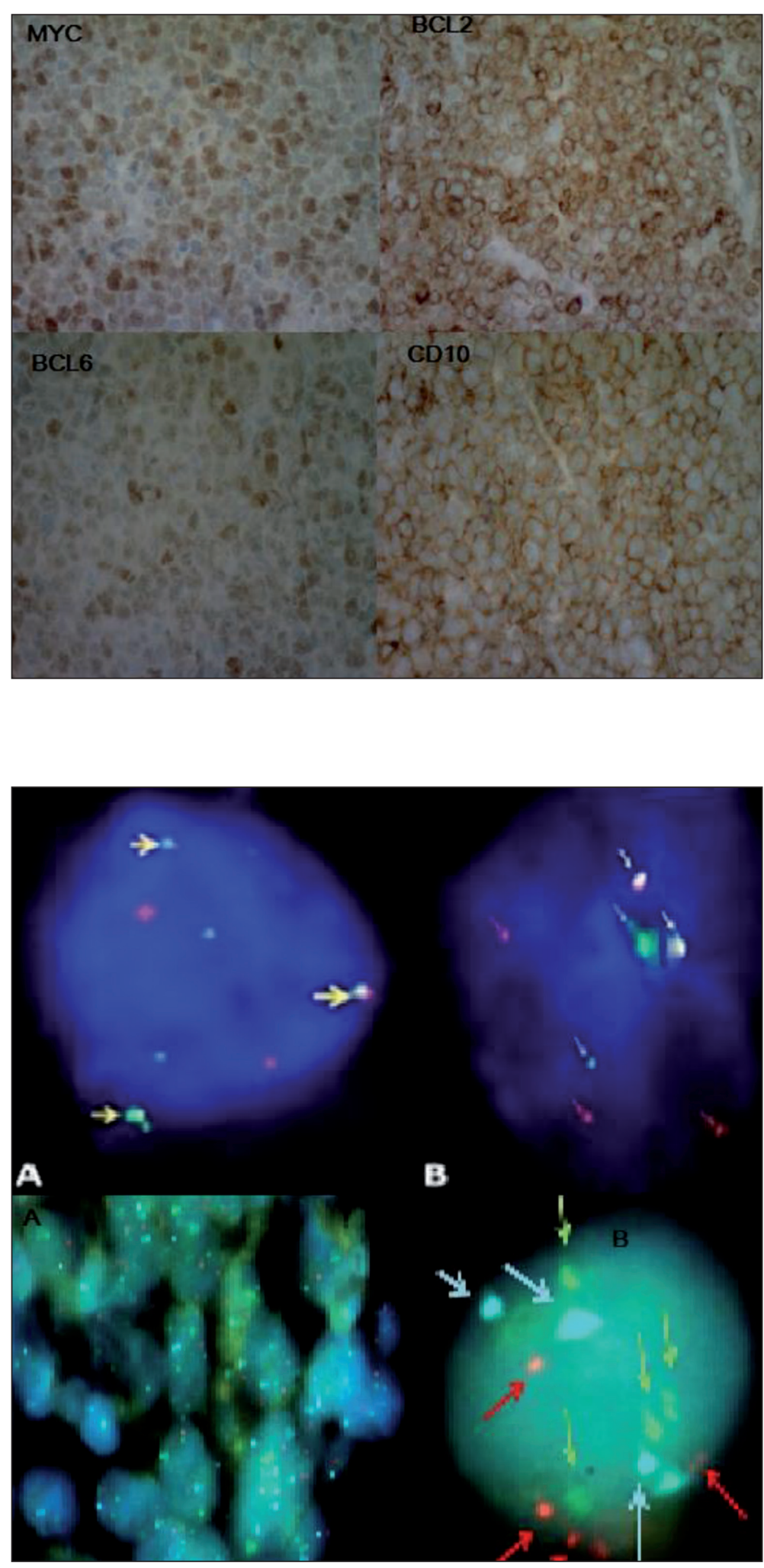

Figura 2. Patrones por inmunohistoquímica del ganglio de la paciente en 40X.
Figura 3. Arriba: FISH para MYC realizado en ganglio de la paciente. A. MYC positivo, patrón 3F2V2R. B MYC positivo, patrón 2F2V3R. Abajo: Patrones de FISH para IGH/MYC/CEP8. A. Se observa ausencia de la traslocación recíproca del gen MYC con IGH (descarta Burkitt). Sin embargo, se observan múltiples patrones en el tejido. B. Detalle de una célula con patrón 3A5R4V. 
rituximab, por lo que debió suspenderse. Evolucionó con compromiso de conciencia, por lo que se realizó TAC cerebro que mostró gran masa cerebral, con gran edema perilesional. La paciente evolucionó en malas condiciones, falleciendo a las 3 semanas de la quimioterapia.

\section{Discusión}

El linfoma difuso de células grandes B (LDCGB) es el linfoma no Hodgkin más frecuente, representando 30 a $40 \%$ según la literatura ${ }^{2}$. En Chile representa 38,5\%, según una revisión local de Cabrera et $\mathrm{al}^{3}$. Dado que el LDCGB es una entidad muy heterogénea en cuanto a morfología, genética y curso clínico, se ha intentado buscar marcadores que logren identificar ciertos grupos con peor pronóstico. Es así como en un comienzo las miradas se enfocaron en los LDCGB con expresión de MYC. Aunque típicamente este se asocia a linfoma de Burkitt (LB), aproximadamente 15\% de los LDCGB reportan traslocaciones relacionadas con MYC, y en 19 a 38\% se reportan ganancias de este ${ }^{4}$. En un comienzo se asoció una peor evolución a la presencia del MYC por sí solo, pero los estudios resultaron inconsistentes. Más tarde, se observó que el mal pronóstico se encontraba en los pacientes que, además de MYC (más de $40 \%$ de positividad), tenían reordenamientos del BCL-2, BCL-6 o ambos $^{5-7}$, por IHQ. Se les denominó "doble o triple expresores" (DE o TE) a estas alteraciones y se encontró en varios estudios peor pronóstico en pacientes tratados con RCHOP. Sin embargo, más recientemente se ha encontrado que los pacientes con mayor correlación con una peor sobrevida son los llamados doble o triple hit (DH o TH), que son las alteraciones encontradas por FISH. Estos responden mal incluso a quimioterapia intensiva tipo R-Hyper CVAD/MTX Ara-C en dosis altas ${ }^{8}$. Nuestro caso tiene ambos estudios con positivos, destacando las alteraciones descritas al FISH, con evolución clonal. No tenemos conocimiento de otro caso con estas características.

Desde 2016, se les denomina linfomas de células B de alto grado, con reordenamiento de MYC, BCL-2 y/o BCL-6, y es una entidad propuesta en la nueva clasificación de neoplasia linfoides de la $\mathrm{OMS}^{1}$. DH y TH representan 3 a $6 \%$ de los LDCGB y conllevan, como ya se mencionó, típicamente mal pronóstico ${ }^{5,69,10}$.

A diferencia de los LB, que también tienen reordenamiento de MYC, estos linfomas se presentan con BCL-2 positivo y cariotipos complejos, como lo observado en nuestra paciente $e^{4,11}$, con múltiples alteraciones estructurales y numéricas. Cabe destacar que además el FISH específico para la $\mathrm{t}(8 ; 14)(\mathrm{q} 34 ; \mathrm{q} 32)$.

Se ha documentado que estos linfomas de biología agresiva se presentan con mayor número de sitios extranodales, compromiso de médula ósea y sistema nervioso central, lo que pudimos observar en nuestro caso. La edad media es de 60 años, mayor que la de nuestra paciente. Es mayormente observado en varones (V2:1M). La mayoría muestra agresividad y alta tasa de recambio, con mayor porcentaje de síntomas B o LDH elevada, lo que también observamos. En cuanto a KI 67, la mayoría muestra 80 a $90 \%$ y la mayoría tiene origen centro germinal, tal como ocurrióo ${ }^{12-14}$.

Sin embargo, observamos algunas características no descritas previamente en estos pacientes. Nuestra paciente se presentó con leucemización del linfoma, raramente reportado en cualquier linfoma $\mathrm{DCGB}^{15}$. No se encontró datos acerca de la relación de hipercalcemia y TH. Hay algunos reportes en linfoma DH transformado desde folicular ${ }^{16}$. En general, la hipercalcemia se presenta en $10 \%$ de los linfomas, y por sí solo representa peor pronóstico. En cuanto al peak monoclonal, que aparece en 10\% de DCGB, también se ha asociado a un peor pronóstico, en especial los IgM. Lamentablemente, no se realizó inmunofijación ni medición de cadenas livianas libres en suero en nuestra paciente, para una mejor caracterización del componente monoclonal.

Si bien pueden ser sensibles a quimioterapia, tienen muy alta tasa de recaída. La sobrevida con RCHOP es menor de $1 \mathrm{año}^{17}$. No existen estudios prospectivos que demuestren cuál es el mejor tratamiento, pero se recomienda regímenes agresivos, tipo Hypercvad, DAepochR, con quimioterapia intratecal ${ }^{18,19}$. El rol del trasplante autólogo es aún controversial $^{20}$.

Concluimos que es un interesante caso que representa muy bien este tipo de linfomas, tanto por su biología como por su agresiva clínica. Es interesante destacar la leucemización de este caso, y su asociación con peak monoclonal e hipercalcemia, además, la descripción de evolución clonal observada al FISH. Todas estas alteraciones se asocian a mal pronóstico por sí solos, pero raramente se presentan en conjunto, y no encontramos otro 
caso con estas alteraciones asociadas a un linfoma TH. Según nuestro conocimiento es el primer linfoma TH reportado de nuestro país.

\section{Referencias}

1. Swerdlow SH, Campo E, Pileri SA, Harris NL, Stein H, Siebert R, et al. The 2016 revision of the World Health Organization classification of lymphoid neoplasms. Blood 2016; 127 (20): 2375-90.

2. Swerdlow SH, Campo E, Harris NL, Jaffe ES, Pileri SA, Stein H, et al. Diffuse large B-cell lymphoma, not otherwise specified. In: WHO Classification of Tumours of Haematopoietic and Lymphoid Tissues. IARC Press: Lyon, France, 2008; 233-7.

3. Cabrera ME, Martínez V, Nathwani BN, Muller-Hermelink HK, Diebold J, Maclennan KA, et al. Non-Hodgkin lymphoma in Chile: a review of 207 consecutive adult cases by a panel of five expert hematopathologists. Leuk Lymphoma 2012; 53 (7): 1311-7.

4. Ott G, Rosenwald A, Campo E. Understanding MYC-driven aggressive B-cell lymphomas: pathogenesis and classification. Blood 2013; 122: 3884-91.

5. Green TM, Young KH, Visco C, Xu-Monette ZY, Orazi A, Go RS, et al. Immunohistochemical double-hit score is a strong predictor of outcome in patients with diffuse large B-cell lymphoma treated with rituximab plus cyclophosphamide, doxorubicin, vincristine, and prednisone. J Clin Oncol 2012; 30: 3460-7.

6. Johnson NA, Slack GW, Savage KJ, Connors JM, Ben-Neriah S, Rogic S, et al. Concurrent expression of MYC and BCL2 in diffuse large B-cell lymphoma treated with rituximab plus cyclophosphamide, doxorubicin, vincristine, and prednisone. J Clin Oncol 2012; 30: 3452-9.

7. Barrans S, Crouch S, Smith A, Turner K, Owen R, Patmore R, et al. Rearrangement of MYC is associated with poor prognosis in patients with diffuse large B-cell lymphoma treated in the era of rituximab. J Clin Oncol 28: 3360-2010.

8. Nowakowski GS, Czuczman MS. ABC, GCB, and Double-Hit Diffuse Large B-Cell Lymphoma: Does Subtype Make a Difference in Therapy Selection? Am Soc Clin Oncol Educ Book. 2015: e449-57.

9. Wang XJ, Medeiros LJ, Lin P, Yin CC, Hu S, Thompson MA, Li S. MYC cytogenetic status correlates with expression and has prognostic significance in patients with MYC/BCL2 protein double-positive diffuse large B-cell lymphoma. Am J Surg Pathol 2015; 39 (9): 1250-8.

10. Hu S, Xu-Monette ZY, Tzankov A, Green T, Wu L, Balasubramanyam A, et al. MYC/BCL2 protein coex- pression contributes to the inferior survival of activated B-cell subtype of diffuse large B-cell lymphoma and demonstrates high-risk gene expression signatures: a report from The International DLBCL Rituximab-CHOP Consortium Program. Blood 2013; 121: 4021-31.

11. Sewastianik T, Prochorec-Sobieszek M, Chapuy B, Juszczyński P. MYC deregulation in lymphoid tumors: Molecular mechanisms, clinical consequences and therapeutic implications. Biochim Biochim Biophys Acta 2014; 1846; 457-67.

12. Snuderl M, Kolman OK, Chen YB, Hsu JJ, Ackerman $\mathrm{AM}, \mathrm{Dal}$ Cin P, et al. B-cell lymphomas with concurrent IGH-BCL2 and MYC rearrangements are aggressive neoplasms with clinical and pathologic features distinct from Burkitt lymphoma and diffuse large B-cell lymphoma. Am J Surg Pathol 2010; 34 (3): 327-40.

13. Yoshida M, Ichikawa A, Miyoshi H, Kiyasu J, Kimura $\mathrm{Y}$, Arakawa F, et al. Clinicopathological features of double-hit B-cell lymphomas with MYC and BCL2, BCL6 or CCND1 rearrangements. Pathol Int 2015; 65: 519-27.

14. Akasaka T, Akasaka H, Ueda C, Yonetani N, Maesako Y, Shimizu A, et al. Molecular and clinical features of non-Burkitt's, diffuse large-cell lymphoma of B-cell type associated with the c-MYC/immunoglobulin heavy-chain fusion gene. J Clin Oncol 2000; 18 (3): 510-8.

15. Chen D, Ketterling R. A leukemic presentation of a "triple-hit" lymphoma Blood 2014; 123: 1126.

16. Xu X, Zhang L, Wang Y, Zhang Q, Zhang L, Sun B, et al. Double-hit and triple-hit lymphomas arising from follicular lymphoma following acquisition of MYC: report of two cases and literature review Int J Clin Exp Pathol 2013; 6 (4): 788-94.

17. Petrich AM, Gandhi M, Jovanovic B, Castillo JJ, Rajguru $\mathrm{S}$, Yang DT, et al. Impact of induction régimen and stem cell transplantation on outcomes in double-hit lymphoma: A multicenter retrospective analysis. Blood 2014; 124: 2354-61.

18. Oki Y, Noorani M, Lin P, Davis RE, Neelapu SS, Ma L, et al. Double hit lymphoma: the MD Anderson Cancer Center clinical experience. Br J Haematol 2014; 166: 891-901.

19. Johnson NA, Savage KJ, Ludkovski O, Ben-Neriah S, Woods R, Steidl C, et al. Lymphomas with concurrent BCL2 and MYC translocations: the critical factors associated with survival. Blood. 2009; 114: 2273-9.

20. Howlett C, Snedecor SJ, Landsburg DJ, Svoboda J, Chong EA, Schuster SJ, et al. Front-line, dose-escalated immunochemotherapy is associated with a significant progression-free survival advantage in patients with double-hit lymphomas: a systematic review and meta-analysis. Br J Haematol 2015; 170 (4): 504-14. 